\title{
Influence of Microstructure on the Magnetic Properties of Goethite ( $\alpha-\mathrm{FeOOH})$
}

\author{
D.F. Valezi ${ }^{1}$, J.C. Spadotto ${ }^{2}$, L.G. Santos ${ }^{1}$, J.P.T. Baú ${ }^{3}$, C.E.A. Carneiro ${ }^{4}$, D. A. M. Zaia ${ }^{3}$, A.C.S. Costa ${ }^{5}$, \\ I.G. Solórzano ${ }^{2}$, E. Di Mauro ${ }^{1}$ \\ 1. Department of Physics, State University of Londrina, Londrina, Brazil \\ 2. Department of Chemical and Materials Engineering, PUC-Rio, Rio de Janeiro, Brazil \\ 3. Department of Chemistry, State University of Londrina, Londrina, Brazil \\ 4. Department of Chemistry, Western Bahia Federal University, Barreiras, Brazil \\ 5. Department of Agronomy, State University of Maringá, Maringá, Brazil
}

Goethite $(\alpha-\mathrm{FeOOH})$ is an iron oxyhydroxide, one of the most abundant mineral in our planet [1]. Despite being a very well-known material, studied for at least half of a century, goethite still has some magnetic properties that are not fully understood. At room temperature, goethite is considered as antiferromagnetic (AFM). However, several studies show that goethite has a magnetic component and relaxation effects, as it can be seen by Electron Paramagnetic Resonance (EPR) [2]. Among many studies on this subject, we cite the Superferromagnetism model (SFM) [3,4]. In this model, the relaxation effects could arise from magnetic mismatches caused by defects in the structure as grain boundaries, dislocations and voids in the crystallites [4].

In this work we conducted two different types of synthesis. Both were performed by two modified methods of Schwetmman [1,5]. For GC sample, $\mathrm{FeCl}_{2} \cdot 4 \mathrm{H}_{2} \mathrm{O}$ was dissolved in water, then added $\mathrm{NaHCO}_{3}$ to goethite formation for 1 hour [5]. For GD sample $\mathrm{Fe}(\mathrm{NO})_{3} .9 \mathrm{H}_{2} \mathrm{O}$ was dissolved in water and added $\mathrm{KOH}$ solution; the suspension was left to goethite formation for 52 days at $50^{\circ} \mathrm{C}$ [1]. With this different processing we aim at generating two different goethite samples in terms of imperfections and defects such as grain boundaries, voids and dislocations. In fact, Infra-red (IR) spectra and X-ray diffractogram of those samples are similar.

Transmission electron microscopy (TEM) analysis, in a JEOL 2100F, reveals that both kinds of samples contains particles in rod-like format, as it appears in Figure 1A of GD sample (GC sample have shown similar results in terms of morphology). The atomic resolution image, Fig 1B, obtained from region indicated by the red box in Figure 1A shows the tip of superimposed rods. Most detailed analysis shows some narrow disordered regions, perhaps a low angle grain boundary, as indicated by arrows. In this HRTEM image the distance between the lattice fringes was measured as $0.25 \mathrm{~nm}$. High resolution images, shown in Figure 2, indicate that the surface of some rods is not smooth but show atomic-scale steps. While GD rods exhibit a slight surface roughness (Figure 2A), the occurrence of these irregularities in GC is more pronounced, presenting a surface containing well-defined steps (Figure 2B).

EPR analysis performed on a JEOL (JES-PE-3X) spectrometer operating in X-band ( 9.5 GHz), have shown resonance lines characteristics of goethite [2]. However, the area over the resonance signal is at least 20 times greater in GC sample than in GD. This observation is in agreement with more pronounced degree of imperfections at GC, because some kind of imperfections can generate non-compensated moments [4]. These subjects are currently under investigations [6]. 


\section{References}

[1] R. M. Cornell, U. Schwertmann. "The iron oxides", ed. 2 (WILEY-VCH, Weinheim).

[2] D. F. Valezi et al, Materials Chemistry and Physics 173 (2016), p. 179-185.

[3] S. Mørup et al, Journal of Magnetism and Magnetic Materials 40 (1983), p. 163-174.

[4] D. E. Madsen et al, Journal of Physics: Condensed Matter 21 (2009), p. 016007 (11p).

[5] U. Schwertmann et al. A lecture and demonstration for students on iron oxide formation. In: 10th Int Clay Conf Clays Controlling the Environment. 1993. p. 18-23.

[6] The authors acknowledge are grateful to CBPF for the access to the LabNano Electron Microscopy facilities and to the Brazilian Funding agencies CAPES and CNPq.
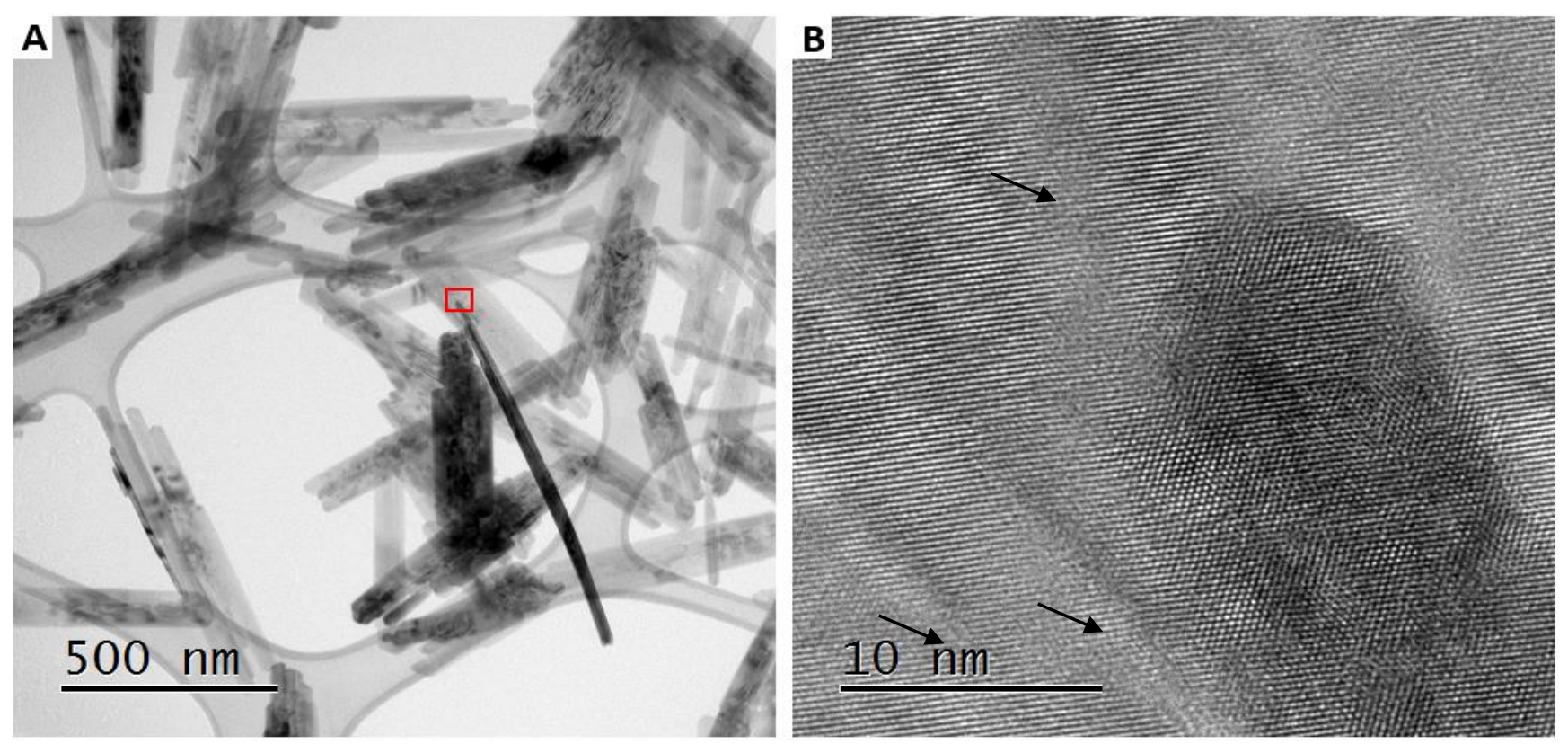

Figure 1. TEM images of GD goethite particles. (A) Bright field TEM image of goethite nanorods and (B) HRTEM image of the boxed area region marked in (A).
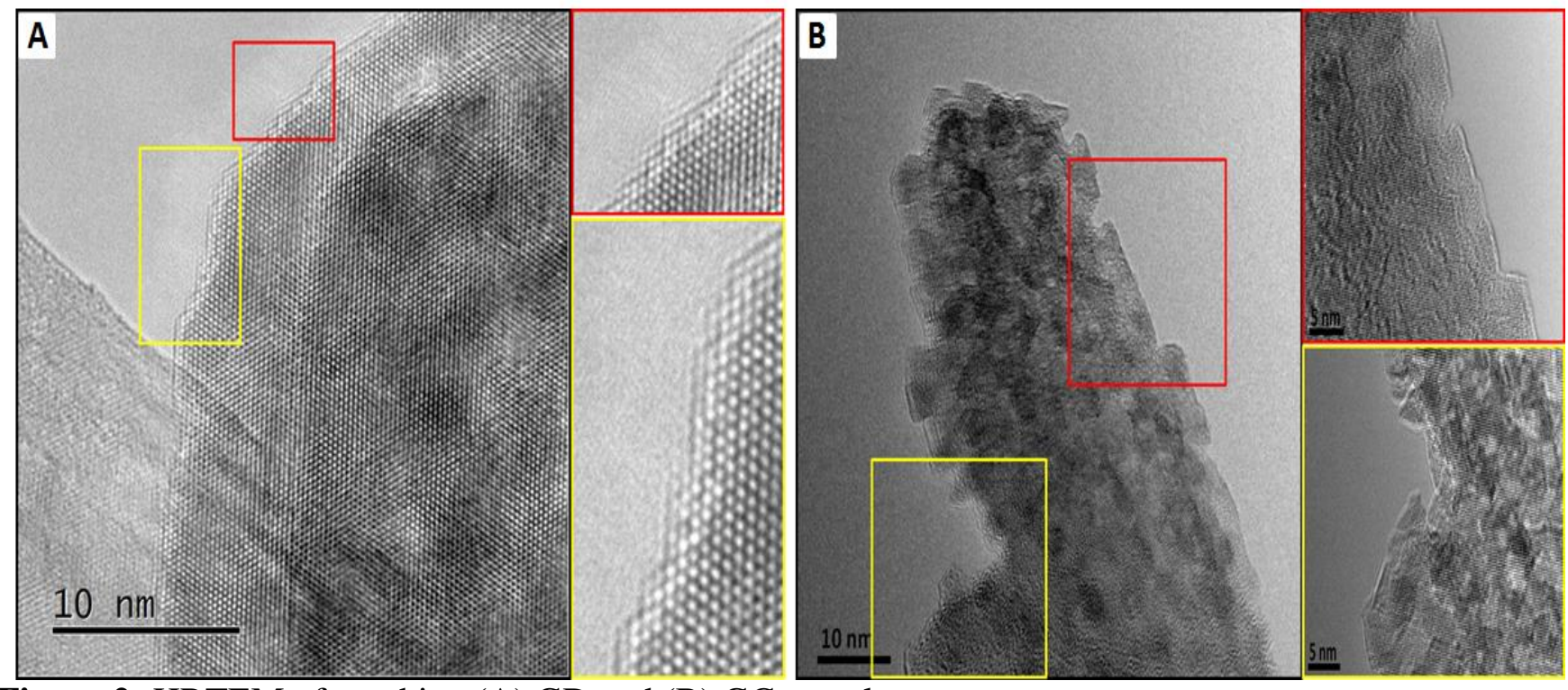

Figure 2: HRTEM of goethite, (A) GD and (B) GC samples. 